\title{
How to Construct Secure Cryptographic Location-Based Services
}

\author{
Jun Anzai ${ }^{1,2}$ and Tsutomu Matsumoto ${ }^{1}$ \\ ${ }^{1}$ Yokohama National University, 79-5, Tokiwadai, Hodogaya, Yokohama 240-8501, Japan \\ \{anzai, tsutomu\}@mlab.jks.ynu.ac.jp \\ ${ }^{2}$ Panasonic Mobile Communications Co., Ltd., 600, Saedo-cho, \\ Tsuzuki-ku, Yokohama 224-8539, Japan \\ anzai.jun@jp.panasonic.com
}

\begin{abstract}
Recently, ubiquitous computing / networks have been studied actively. These networks provide services depending on real environments of mobile nodes. Especially, we expect location-based services (LBSs), which rely on location of mobile nodes, are anticipated to come into wide use in the future. High-value LBSs require cryptography to ensure security. Here, cryptographic LBSs comprise a key management function (e.g. key sharing with nodes) and a location management function (e.g. location verification of nodes). Cooperation between key and location management functions realizes cryptographic LBSs. However, these functions have mostly been studied individually. This study indicates that cryptographic LBSs are insecure if the cooperation is incomplete, and proposes a method of constructing secure cryptographic LBSs.
\end{abstract}

\section{Introduction}

\subsection{Background}

Recently, services using the real context of mobile nodes are actively studied on ubiquitous computing. We expect services, which use location information of nodes as a real context, to come into wider use in the future. Such services are called locationbased services (LBSs). LBSs include walker navigation, mobile node tracking [9] [15], location-based access control [6], along with other applications. Many highvalue LBSs require security; for that reason, LBSs require cryptographic capability. As instances of cryptographic LBSs, we postulate a system in which a user can read a secret business document stored in a notebook PC in an office. In this system, reencryption of the document prevents its reading when the PC is removed from the office. Cryptographic LBSs comprise a key management function (e.g. key sharing for session encryption) and a location management function (e.g. node location verification). Key management methods have been studied variously until now. As location management, location measurement methods using global position system (GPS) and radar are realized; methods using wireless LAN and radio frequency identification (RFID) are advancing apace. However, these management methods have mostly been studied individually. Therefore we consider security of integration between key management and location management for realizing secure cryptographic LBSs. 


\subsection{Location Management}

Location measurement technologies have made the transition from methods [7] using GPS, a base station (of cellular phones) and radar for outdoors to methods [10][11] [13] using wireless LAN, RFID for indoors. Papers [2][3][5][12][14] proposed secure location verification schemes using communication delay. This paper refers to digitized location information as a "Location Token". We assume a location token model that composes plural location token providers (with various location measurement functions), provers (which prove their own location using the tokens) and verifiers (which verify prover location using the tokens). One study [14] adopts a narrowlydefined location token mode that supposes one location measurement scheme. This paper uses location management as a generic term to refer to the following functions:

- Location verification: a verifier directly verifies a mobile node location in realtime.

- Location certification: a verifier indirectly verifies a mobile node location using location tokens.

\subsection{Key Management}

In this paper, key-management targets are the following keys: a client private key: is a unique secret key of a mobile node (and the corresponding public key), and a processed key: is output of a key-management function that is an inputted client private key dependent on information, the output is a secret key (and the corresponding public key). Key management is a generic term used to refer to the following functions:

- Key issuing: is a method that issues a key, e.g., issuance of a public key certificate;

- Key sharing: is a method by which plural entities share the same key, for instance Diffie-Hellman key exchange scheme;

- Key distribution: is a method that distributes a key to specific entities, for instance broadcast encryption,

- Key generation: is a method that one or more entities generate a key, for instance RSA key generation,

- Key revocation: is a method that revokes a key, for instance broadcast exclusion and Certificate Revocation List; and

- Key control: is a method that controls access to a key, for instance Kerberos.

\subsection{Integration of Key Management and Location Management}

One study [6] proposed a PC system, in which a PC hard disk is decrypted because a personal radio device allows the PC to use a decryption key if their authentication is successful, when the device closes in the PC. Another study [4] proposed trusted access points measuring mobile node location and shares a key using electric field intensity of beacons sent by the points. The studies are schemes that mix location management and key management functions. However the studies do not clarify the structure of integration between the two functions. Thus we cannot analyze structural security. Incomplete integration might cause an attack on cryptographic LBSs, e.g., a provider would like to share a key with a mobile node on a specific location. However, an attacker on other location may force the provider to share the key with the at- 
tacker after the valid node location verification. Therefore, we consider security of integration between key management and location management for realizing secure cryptographic LBSs. This paper treats three problems: 1) an attacker may impersonate a valid node if target nodes are not the same on key management and location management functions; 2) an attacker may replace a valid function with an invalid function if key management and location management functions are indivisible; and 3) a provider may not provide valid LBSs if a function execute after execution of another function is a failure.

\subsection{Our Goal}

Cryptographic LBSs require key management and location management functions. Secure integration of these functions has not clarified because the functions have only studied individually until now. This paper proposes a method of integrating key management and location management functions for realizing secure cryptographic LBSs. In addition, we suggest new cryptographic LBSs by assessing combinations of key management and location management. Our proposed method defines two general integrated functions (location key function). Our method consists of a location key server that provides services using location key functions and a location key client that requests a service to the server. For realizing secure cryptographic LBSs, we apply approaches: 1) agreement of target nodes by a context connection (CC) value; 2) improvement of mutual dependence by a construct inputs output of a management function to another management function; and 3) policy-based access control for functions.

\section{Definitions and Requirements}

\subsection{Location Key}

In this paper, a location key function means an integrated function of key management and location management functions. The location key function is classified as follows:

- LK (Location operation, then Key operation) function - is a key management function that is inputted to output of a location management function; and

- KL (Key operation, then Location operation) function - is a location management function that is inputted to output of a key management function.

LK and KL functions output a pair of a location key and its corresponding location token. Note that an LK function does not output a location token basically. A location key is a processed key: that is outputted from an LK function; or is targeted by a location token. The location keys are determined from location between a client location, a time when location key function is executed, and a client private key.

\subsection{Entities}

Our proposed method consists of the following entities:

- Location key client: is a mobile node that requests services using location key functions to a location key server. A client stores a unique client private key se- 
curely and his ID is "i". In addition, the client might obtain his own location information, time information, and random numbers. This study supposes a cellular phone, PDA and a notebook PC as clients.

- Location key server: is a server that provides services using location key functions to a location key client. A server has KL and LK functions as location key functions, and stores a unique server private key securely; the ID is " $\mathrm{j}$ ". The server provides services using the location key functions according to a location key policy to a location key client. A server might obtain its own location information, time information, and random numbers. This study supposes the following as servers: 1) Station: is a trusted apparatus that has high performance and is fixed on a specific location. This study presumes a base station of cellular phone systems and an access point (or a PC connecting to the point) of a wireless LAN as a station; 2) Mobile: is a mobile node that has middle performance. This study supposes a notebook PC, a PDA, or a cellular phone as a mobile. A location key client does not necessarily trust a mobile; 3) Sensor: is a fixed node that has low performance and is active. This study supposes a sensor node of sensor networks and an active IC tag (e.g. a smart tag) of RFID as a sensor. A sensor is not highly trusted by a location key client; 4) Tag: is a device that has little performance and is passive. This study supposes a tag of RFID as a tag. A location key client does not completely trust. In case of wearing a tag, a reader/writer writes a location token to the tag. Therefore, the tag is a location key client and the reader/writer is a location key server.

\subsection{Location Measurement Methods}

We classify methods that measure client locations into the following types: 1) Report type: means a method in which a client reports self-obtained location of the client to a server. This study presumes a client supporting system, for example GPS; 2) Inference type: means a method in which a server infers location of a client from evidence (e.g. IDs of tags). This study generally presumes methods using RFID; 3) Direct type: means a method in which a server directly verifies location of a client in real-time. This study presumes methods using radar, a wireless LAN, and communication delay.

\subsection{Location Token}

A location token is digitized location information obtained from a location key server with location measurement methods. The token includes a location key client ID, client location information and a time when a location key function executes. The token also includes a location key, or a location key function issues a pair of a location token and the corresponding location key. This study assumes the following location tokens.

- A location certificate: is a kind of public key certificate with which a station or a mobile signs IDs of a client and a server; client location information, a location key and a time when a location key function executes. Apparently, a location certificate is a kind of time-stamp [8] that includes location information and a location key. The location certificate supposes direct type location measurement methods.

- Location evidence: is digitized location information with a message authentication code that a location key client obtains from a sensor. Use of location evidence supposes inference-type location measurement methods. The location evidence in- 
cludes a sensor ID or client location information. The location evidence might include a client ID, a location key and a time when a location key function executes.

- Provisional location evidence: is digitized location information that a location key client obtains from a tag. The provisional location evidence supposes inferencetype location measurement methods and includes a tag ID.

- A location reference: is digitized location information that a location key client self-calculates using supporting entities (e.g. GPS). The location reference supposes report-type location measurement methods.

Table 1 show the relation between location tokens, servers and location measurement methods. A hyphen means that the corresponding method is nonexistent now. Define the corresponding new location token if a method that corresponds to the hyphen appears. A station, a mobile and a sensor receive a location reference and can then transform the location reference to a location certificate or evidence.

Table 1. Relation between location tokens, servers and location measurement methods

\begin{tabular}{|l|l|l|l|}
\hline $\begin{array}{l}\text { Method } \\
\text { Server }\end{array}$ & $\begin{array}{l}\text { Report type meas- } \\
\text { urement }\end{array}$ & Inference type measurement & $\begin{array}{l}\text { Direct type meas- } \\
\text { urement }\end{array}$ \\
\hline Station & Location reference & - & Location certificate \\
\hline Mobile & Location reference & - & Location certificate \\
\hline Sensor & Location reference & Location evidence & - \\
\hline Tag & - & Provisional location evidence & - \\
\hline
\end{tabular}

\subsection{Security Assumptions}

This paper makes the following security assumptions:

1. A location key server becomes trust, a station, a mobile, a sensor and a tag in that order. Especially the station is a trusted party.

2. Each key management method and each location management method is secure.

3. An attacker is a location key client or a third party.

4. For attacking, a location key client and a third party might conspire.

5. A communication channel is not secure: anyone can obtain data on the channel.

6. An attacker purposes location key functions of a location key server to use illegally, and purposes outputs of the location key functions to use change illegally.

\subsection{Requirement}

This paper designs our proposed method for satisfying the following requirements:

1. Availability: is that only allowed location key clients can use location key functions of a location key server according to a location key policy.

2. Universality: is that our method is easily adaptable to existing systems. Actual systems, which include various servers, location measurement technologies, key and location management methods, require our method to universal design.

3. Associativity: is that association between a key management function and a location management function is secure. Consequently, our proposed method solves three problems shown in section 1.4. 
4. Privacy: is that information (a client ID, client location information and a time when a location key function executes), which is demanded by a client demands to conceal, is not leaked from a location token. A server excludes inconsistent requests of a client. This paper respectively refers to concealing an ID, concealing client location information, and concealing a time when a location key function executes "anonymity, location-hiding and time-hiding" respectively.

\section{Proposed Method}

\subsection{Notation}

We next show the notation for explaining our proposed method:

- CK: is a client private key. SK: is a server private key. $\mathbf{R}$ : is a random number.

- CID: is an ID of a location key client. SID: is an ID of a location key server.

- Info $\mathbf{C}_{\mathbf{C}}$ : is location key client information that consisting of $\mathrm{R}$, location information and time information. Infos: is location key server information that comprising of $\mathrm{R}$, location information, and time information.

- Data $_{\mathbf{C K}}$ : is data that depends on CK (and Info $_{\mathrm{C}}$ ). PK: is a processed key that is the output of a key management function with input is Data ${ }_{\mathrm{CK}}$.

- LKP: is a location key policy that includes conditions for executing location key functions. LKS: is an internal status of a location key server: the status (e.g. existence of a specific key, and current location of a server) is needed for judging LKP.

- KMT: is a type of key management (key issuing, key generation, key sharing, key distribution, key revocation, key control and none). LMT: is a type of location management (location verification, location certification and none). LKT: is a type of location key function (KMT \| LMT and LMT || KMT).

- LTT: is a type of location token (a location certificate, location evidence, a location reference, and none). CPT: is a type of client privacy (anonymity, locationhiding, time-hiding, and none).

- CC value: is a context connection value (CID, $\mathrm{R}, \mathrm{PK}$, or location token).

- KM function: is a key management function that outputs PK for input (Data $\mathrm{C}_{\mathrm{CK}}$, KMT, CID, SID, SK, Info ${ }_{C}$, and Info $_{S}$ ). According to KMT, \{CID, SID, SK, Info and Info $\left._{S}\right\}$ cannot be ignored. As PK, a KM function outputs CID (in case that KMT indicates a key management method with client authentication) or R (in case

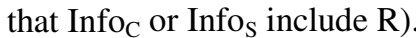

- LM function: is a location management function that outputs a location token for input (Data ${ }_{\mathrm{CK}}$, LMT, SID, SK, Info ${ }_{\mathrm{C}}$ Info $_{\mathrm{S}}$, and LTT). According to LMT, \{CID, SID, SK, Info ${ }_{\mathrm{C}}$ and $\left.\mathrm{Info}_{\mathrm{S}}\right\}$ cannot be ignored. The LM function outputs a preselected location token if LTT is none. As a location token, the LM function outputs CID (in case that LMT indicates a location management method with client

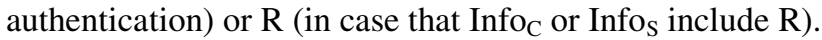

- PJ function: is a policy judgment function that outputs KMT, a pair of \{CPT, LMT, LTT $\}$ or Reject for input (LKP, CPT, LTT, LKS, LKT, CID, and Info $_{\mathrm{C}}$ ) According to LKP, $\left\{\right.$ CPT, LTT, LKS, LKT, CID, and Info $\left._{\mathrm{C}}\right\}$ cannot be ignored.

- KL function: is a location key function that outputs a pair of \{a location key, a location token or Reject for input $\left(\right.$ Data $_{\mathrm{CK}}, \mathrm{LKP}, \mathrm{LKS}, \mathrm{LKT}, \mathrm{CID}, \mathrm{SID}, \mathrm{SK}, \mathrm{Info}_{\mathrm{C}}$, 
Info $_{S}$, LTT, and CPT). A location token includes a location key if LTT = "location certificate". According to LKT and LKP, $\left\{\mathrm{LKS}, \mathrm{LKT}, \mathrm{CID}, \mathrm{SID}, \mathrm{SK}\right.$, Info $_{\mathrm{C}}$, Info $_{\mathrm{S}}$, LTT, and CPT \} cannot be ignored.

- LK function: is a location key function that outputs a location key or Reject for input $\left(\right.$ Data $_{\mathrm{CK}}$, LKP, LKS, LKT, CID, SID, SK, Info ${ }_{\mathrm{C}}$, and Info $_{\mathrm{S}}$ ) According to LKT and LKP, $\left\{\right.$ LKS, LKT, CID, SID, SK, Info ${ }_{\mathrm{C}}$ and Info $\left._{\mathrm{S}}\right\}$ cannot be ignored.

\subsection{Constructions of a KL Function and an LK Function}

Figure 1 shows constructions of KL and LK functions that comprise KM, LM and PJ functions. The KL function consists of a management path that inputs output of a KM function into an LM function, and a control path that inputs output of a PJ function into KM and LM functions. On the management path, a KM function executes a key operation, which is requested by a key management type KMT, with a location key client for input (a client ID CID, a server private key SK, server information Info $_{S}$, a server ID SID, and client information $\operatorname{Info}_{\mathrm{C}}$ ); and then the KM function outputs a processed key PK. In addition, the KM function outputs CID (if the KM function performs client authentication) or a random number $\mathrm{R}$ (if the KM function performs client distinguishing with a temporal ID (i.e. R)) as $\mathrm{CC}$ values. The $\mathrm{PK}$ is a $\mathrm{CC}$ value if the KM function does not output CID or R. Next, an LM function executes a CC value-dependent location operation, which is requested by a location management type LMT, with a client for the output of the KM function, and then the LM function outputs a pair of $\{$ a location key, a location token $\}$ that is requested by a client privacy type CPT and a location token type LTT. Here, a term "CC value-dependent" means that the LM function authenticates that the client has CID, R or the corresponding secret information. On the control path, a PJ function controls execution of a KM function according to a location key policy LKP, a location key status LKS and Request of a client. Next, the PJ function controls execution of an LM function according to the output of the KM function, LKP, LKS, and the Request. If the PJ function outputs Reject, the KL function stops execution and outputs Reject. The LK function consists of a management path that inputs output of an LM function into a KM function and a control path that inputs output of a PJ function into LM and KM functions.

On the management path, an LM function executes location operation, which is requested by LMT, with a client for input (CID, SK, Info ${ }_{S}$, SID and Info $_{C}$ ); and then the LM function outputs a pre-selected location token. The LM function outputs CID (if the LM function performs client authentication) or R (if the LM function performs client distinguishing with a temporal ID (i.e. R)) as CC values. The location token is a $\mathrm{CC}$ value if the LM function does not output CID or R. Next, a KM function executes a $\mathrm{CC}$ value-dependent key operation, which is requested by KMT, with a client for the output of the LM function, and then the KM function outputs a location key. On the control path, a PJ function controls execution of an LM function according to LKP, LKS and the Request of a client. Here, the PJ function inputs none as CPT and LTT. Next, the PJ function controls execution of a KM function according to the output of the LM function, LKP, LKS and the Request. If the PJ function outputs Reject, the LK function stops execution and outputs Reject.

Here, LKP comprises plural records. Each record includes three items: an attribute that is client identification or a client belonging, an action that is an allowed location key functions operating, and a condition that is a requirement to allow the action. 
LKP, LKS, ReCID, SID, SK, quest (CID, CPT,

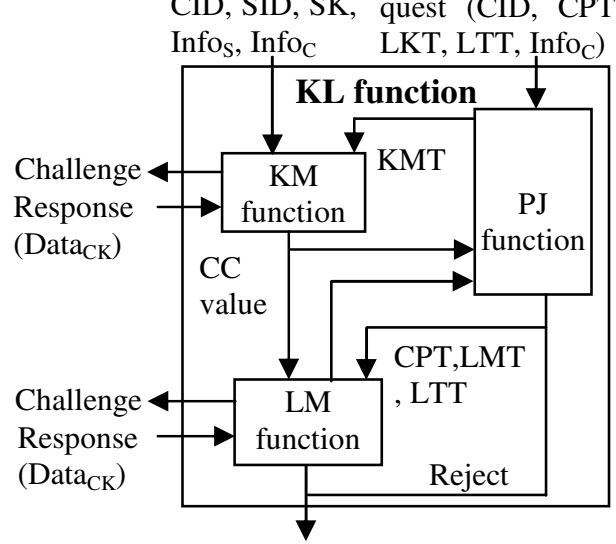

A pair of \{a location key, a location token $\}$, or Reject
LKP, LKS, ReCID, SID, SK, quest (CID, CPT,

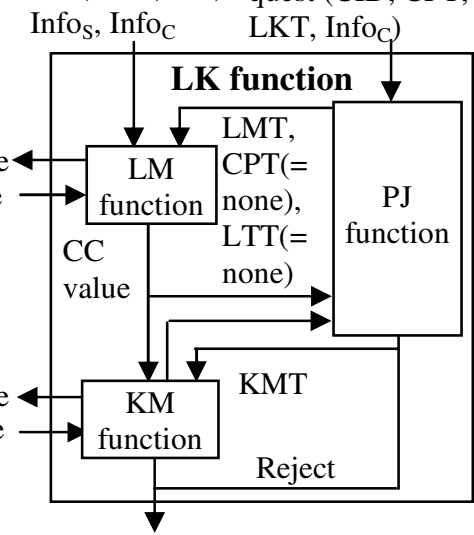

A location key, or Reject

Fig. 1. Constructions of a KL function and an LK function

\subsection{Sequence of Our Proposed Method}

Figure 2 shows a sequence of our proposed method.

\section{Location Key Client i Location Key Server j}

\begin{tabular}{|c|c|c|}
\hline $\begin{array}{l}\text { 1. i sends Request (CID \| } \\
\text { LKT (= KMT \| LMT) \| In- } \\
\text { fo }_{\mathrm{C}}\|\mathrm{CPT}\| \mathrm{LTT} \text {, or CID \| } \\
\text { LKT (= LMT \| KMT) \| } \\
\text { 4. i obtains a pair of \{a loca- } \\
\text { tion key, a location token\}, } \\
\text { a location key or Reject } \\
\text { from Location key message. }\end{array}$ & 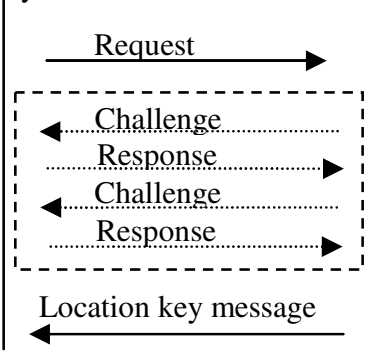 & $\begin{array}{l}\text { 2. j executes a location key } \\
\text { function including KM } \\
\text { and LM functions (e.g. } \\
\text { challenge-response func- } \\
\text { tions) from Request. } \\
\text { 3. j sends Location key } \\
\text { message ((a location } \\
\text { key, a location key), a } \\
\text { location key or Reject). }\end{array}$ \\
\hline
\end{tabular}

Fig. 2. Sequence of our proposed method

\subsection{Combinations of Key Management and Location Management}

We introduce instances of combinations (see Table 2) of key and location management, and believe that other concrete schemes exist in addition to the following instances.

- LK function 2: is a scheme that a server verifies client location and shares a symmetric key with the client if the location is within $50[\mathrm{~m}]$ (e.g. the scheme [4] exists).

- KL function 3: is a group key generation in which group members can certificate who, where, and when to share the key with a third party, using the scheme [1].

- LK function 4: is a scheme by which a server verifies the client location; then the server distributes a decryption key for encrypted data (e.g. business documents) if the location is in the specific area (e.g. an office). For example, scheme [6] exists. 
Table 2. Combinations of key management and location management

\begin{tabular}{|l|l|l|l|l|}
\hline Key Location & Location verification & Location certification \\
\hline Key issuing & KL function 1 & LK function 1 & KL function 7 & LK function 7 \\
\hline Key sharing & KL function 2 & LK function 2 & KL function 8 & LK function 8 \\
\hline Key generation & KL function 3 & LK function 3 & KL function 9 & LK function 9 \\
\hline Key distribution & KL function 4 & LK function 4 & KL function 10 & LK function 10 \\
\hline Key revocation & KL function 5 & LK function 5 & KL function 11 & LK function 11 \\
\hline Key control & KL function 6 & LK function 6 & KL function 12 & LK function 12 \\
\hline
\end{tabular}

\section{Evaluation}

\subsection{Viability of Our Proposed Method}

This section shows that our proposed method satisfies the following requirements.

Availability: A location key server has location key functions; a PJ function can control those functions using a location key policy. Consequently, only the allowed location key client can use the location key functions. Here, a KM function or an LM function (in the location key functions) authenticates client identification.

Universality: As shown in Table 1, our method assumes four location token types that correspond to combinations of four location key server types and three location measurement method types. A location key client can request a provided location token type to the server. Therefore, various existing systems can adopt our method. Moreover, existing key and location management schemes can apply to a KM and an LM functions because our method treats the KM and LM functions as modules.

Associativity: From Figure 1, a KL function structure can force output of a KM function to be input of an LM function for boosting the relation between the KM and LM functions. In the same way, an LK function structure can force output of an LM function to be input of a KM function. On location key functions, KM and LM functions can authenticate the same client using CC values, for preventing differences between clients whom the KM and LM functions authenticate. Here, a CC value (CID, R, PK, and a location token) depends on a client by which each management function targets as follows: 1) a server can identify a client because CID is a unique ID; 2) a server can distinguish a client that has $\mathrm{R}$ from another client that does not have the R. But the server cannot identify a client because the $\mathrm{R}$ is a temporal ID by which the server gives for providing LBSs; 3) a server can distinguish a client that has PK from another client that does not have the PK. But the server may not be able to identify a client because a key management method may not authenticate the client on a KM function; 4) a server can distinguish a client that has a location token from another client that does not have the token. But the server may not be able to identify a client because the server may not identify the client for location management. Thus, a server can authenticate the same client using a CC value when the client requests anonymity on client authentication, and when management methods of LM and KM functions do not support a temporal ID. Here, securities of CC values rely on that of the methods. In addition, location key 
functions inputs are limited to a Request and Responses. A PJ function can verify the Request directly. On the other hand, KM and LM functions verify the Responses; a PJ function verifies feedback from the KM and LM functions. In a word, the PJ function can verify the Responses indirectly. The PJ function can also stop execution of a location key function if the PJ function receives feedback that the KM and LM function are unable to authenticate the same client. Therefore, the PJ function can verify a management path between the KM and LM functions.

Privacy: A server outputs an only location token as client privacy information. A client can demand anonymity, location hiding and time hiding to the server using a CPT. The server generates the token, which is excluded privacy information selected by the CPT, if a PJ function allows the token generation from a LKP.

\subsection{Security Analysis}

We analyze security of our method. From section 2.5 and 4.3, location key functions are secure for external attacks. In addition, a location key server is a trusted party when the server is a station. Consequently, our method can prevent attacks described in section 2.5 if a location key server type is a station. The security of our method is equal to trustiness of a server when the server type is a mobile, a sensor, or a tag.

\section{Conclusion}

This paper proposed a method of constructing secure cryptographic LBSs, which have a location key function consisting mainly of a location management function and key management function. Our proposed method includes three approaches: apply a construct by which output of a management function inputs another management function, context connection value, and policy-based access control to location-key functions.

\section{References}

[1] J. Anzai, T. Matsumoto, "Interaction Key Generation Schemes," IEICE Trans. Fundamentals, Vol. E87-A, No. 1, pp. 152-159, 2004.

[2] J. Anzai, T. Matsumoto, "Location Verification (1): Location Verification Schemes Resistant Against Relay Attack," Proc. of SCIS2005, 2B4-3, 2005.

[3] S. Brands, D. Chaum, "Distance-Bounding Protocols, “ Proc. of Eurocrypto'93, Springer-Verlag, pp. 344-359, 1993.

[4] S.Banerjee, A.Mishra, "Secure Spaces: Location-based Secure Wireless Group Communication," Mobile Computing and Communications Review, Vol. 1, No. 2, 2002.

[5] S. Capkun, J. P. Hubaux, "Securing position and distance verification in wireless networks," Technical report EPFL/IC/200443, 2004.

[6] M.D.Corner, B.D.Noble, "Zero-Interaction Authentication," Proc. of MOBICOM2002.

[7] E. Gabber, A. Wool. "How to prove where you are: Tracking the location of customer equipment," Proc. of 5th ACM Conf. Computer and Communications Security, pp.142149, 1998.

[8] S. Haber, W. S. Stornetta, "How to Time-Stamp a Digital Document," Journal of Cryptology: the International Association for Cryptologic Research 3, 2, 99-111, 1991. 
[9] M. Izumi, S. Takeuchi, Y. Watanabe, K. Uehara, H. Sunahara, J. Murai, “A Proposal on a Privacy Control Method for Geographical Location Information Systems," Proc. of INET2000.

[10] T. Kitasuka, T. Nakanishi, and A. Fukuda, "Indoor Location Sensing Techinique using Wireless Network,” Proc. of Computer System Symposium'02, pp. 83-90, 2002.

[11] K. Nakanishi, J. Nakazawa, and H. Tokuda, "LEXP: Preserving User Privacy and Certifying the Location Information," 2nd Workshop on Security in Ubicomp2003.

[12] N. Sastry, U. Shankar, and D. Wagner, "Secure Verification of Location Claims, " Report No. UCB//CDS-03-1245, University of California, Berkekey.

[13] A.Vora, M.Nesterenko, "Secure Location Verification Using Radio Broadcast," Proc. of OPODIS2004:8th International Conference on Principles of Distributed Systems, 2004.

[14] B. R. Waters, E. W. Felten, "Secure, Private Proofs of Location," Princeton University Computer Science Technical Reports, TR-667-03, 2003.

[15] Y.Watanabe, S.Takeuchi, F.Teraoka, K.Uehara, and J.Murai, "The Geograhical Location Information System with Privacy Protection,” IPSJ Journal, Vol. 37, No. 6, 1996. 